\title{
Effect of Contact Parameters on the Accuracy and Computational Efficiency of Finite Element Analysis in the Interference Analysis
}

\author{
Bharat Panjwani \\ ANSYS Software, Kabra Excelsior, \#6A, 7th Main, 1st block, Koramangala, Bangalore, India
}

\begin{abstract}
Contact pressure generated due to interference is of high interest to simulation engineers in commonly used interference method to assemble components in the industry. FEA (finite element analysis) is widely used to evaluate contact pressure in assemblies thus it is crucial to understand the effect of contact parameters on the accuracy and computational efficiency of FEA. In this study, 3D (three-dimensional) model of concentric cylinders' assembly was analyzed for contact pressure at different radial interference values. The effect of contact parameters (formulation algorithms, stiffness) on the accuracy and computational efficiency was investigated. All analyzed cases provided results close to analytical results though showed variation in the computational efficiency. Later part of this study also examines the accuracy of the user defined surface contact offset method in comparison with geometrical penetration method to simulate interference in FEA.
\end{abstract}

Keywords: Interference, Contact formulations, Finite element analysis, Contact offset

\section{Introduction}

Press fit and shrink fit are widely used in assemblies to avoid free play between components. Interference generates contact pressure and frictional forces at contact interfaces which are important results to assess the integrity and reliability of assemblies in field conditions. If contact pressure is not sufficient, it can lead to disassembly of parts during operation. On the other side, excessive pressure can damage the parts. Contact pressure also affects life of parts by influencing fretting fatigue. These requirements make it critical to evaluate contact pressure accurately.

There are different parameters which can affect contact pressure at interfaces such as penetration, material properties, surface roughness, friction coefficient, lubricant conditions and applied constraints. Analytical results for the calculation of contact pressure in press fit and shrink fit assemblies are only available for simplified cases. Analytical solutions are generally not available for assemblies of interest due to nonlinear conditions at the contact interfaces thus numerical techniques like FEA (finite element analysis) have become popular to analyze practical assemblies of interest. In the literature, various experimental, analytical and numerical methods to calculate contact pressure have been evaluated [1]-[3]. Different studies have been carried out to correlate experimental results with FEA results for interference fitted assemblies [4]-[7].

With ongoing developments in high performance hardware and computational methods, FEA is widely used in industry by simulation engineers to study the effect of interference on assembly conditions. Complex geometries having interference can be analyzed for contact results using FEA to reduce product development time. Effect of contact parameters on press-fit curve has been evaluated in an earlier study [8]. With growing use of FEA, it is important to investigate more about the influence of contact parameters on the accuracy and computational efficiency of results in interference analysis.

In this study, assembly of concentric cylinders with interference is analyzed to study the effect of different contact parameters (formulation algorithms, stiffness) on the accuracy by comparison with the analytical results. Effect of contact parameters on the computational efficiency was also investigated.

Analytical calculations for contact pressure (p) are carried out (1) as per thick walled cylinders theory [9] (refer figure $1)$.

$$
\mathrm{p}=\frac{\delta}{R\left[\frac{1}{E_{\mathrm{o}}}\left(\frac{r_{\mathrm{o}}^{2}+R^{2}}{r_{\mathrm{o}}{ }^{2}-R^{2}}+v_{\mathrm{o}}\right)+\frac{1}{E_{\mathrm{j}}}\left(\frac{R^{2}+r_{\mathrm{i}}^{2}}{R^{2}-r_{\mathrm{i}}{ }^{2}}-v_{\mathrm{i}}\right)\right]}
$$

$\delta=$ Radial Interference

$R=$ Nominal Radius

$E_{\mathrm{c}}=$ Young's Modulus of Outer Cylinder's Material

$E_{1}=$ Young's Modulus of Inner Cylinder's Material

$r_{0}=$ Outside Radius of Outer Cylinder

$r_{1}=$ Inside Radius of Inner Cylinder

$v_{0}=$ Poisson's Ratio of Outer Cylinder's Material

$v_{\mathrm{I}}=$ Poisson's Ratio of Inner Cylinder's Material

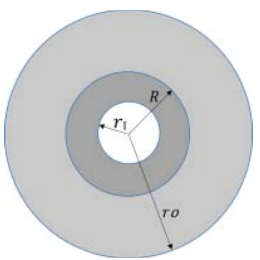

Figure 1: Concentric Cylinders Assembly

In the second part of this study, comparative studies were carried out for different methodologies of modeling assembly interference. In first methodology (referred as geometry based interference), inside radius of outer cylinder was kept higher than outside radius of inner cylinder by 


\section{International Journal of Science and Research (IJSR) \\ ISSN (Online): 2319-7064}

Index Copernicus Value (2013): 6.14 | Impact Factor (2015): 6.391

value equal to the radial interference. In this method, interference was modeled by using suitable geometrical dimensions. In the second methodology (referred as contact offset based interference), inside radius of outer cylinder was kept equal to the outside radius of inner cylinder. Radial interference was modeled by using contact parameter referred as contact offset. In this method, interference is simulated by adjusting contact parameter in FE (finite element) model. Comparative study for the accuracy of contact offset based interference in comparison with geometry based interference was carried out to assess the applicability of each method.

\section{Methodology}

Complete FEA was carried out in ANSYS Workbench. Three-dimensional assembly model of concentric cylinders was set up and analyzed in ANSYS Mechanical R17.0. To take advantage of symmetry, only quarter symmetry model was simulated. Geometry was prepared in DesignModeler as per dimensions (mm) listed in Table 1. Both cylinders were assigned isotropic material properties of structural steel as listed in Table 2.

Table 1: Geometrical Parameters of Assembly

\begin{tabular}{|c|c|}
\hline Outside radius of the outer cylinder & 2000 \\
\hline Inside radius of the outer cylinder & 1000 \\
\hline Outside radius of the inner cylinder & $1000+$ radial interference \\
\hline Inside radius of the inner cylinder & 0 \\
\hline Length of inner and outer cylinders & 100 \\
\hline
\end{tabular}

Table 2: Material Properties

\begin{tabular}{|c|c|}
\hline Young's Modulus & 2E+05 MPa \\
\hline Poisson's Ratio & 0.3 \\
\hline
\end{tabular}

Assembly of concentric cylinders was meshed in ANSYS Workbench using higher order solid (SOLID 186) elements (Node count $\sim 43000$; Element count $\sim 8700$ ). Figure 2 (a) shows meshed regions (thickness not shown). All volumes were meshed using sweep method. Symmetrical boundary conditions were applied on the cut faces of quarter symmetry model as shown in Figure 2 (b). Frictionless support was applied at one end of the cylinders' assembly while another end was kept free.

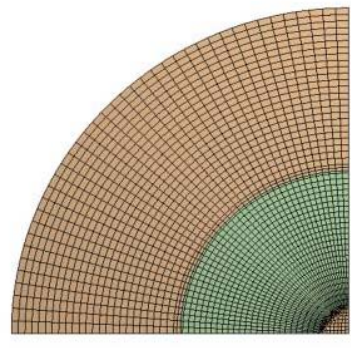

(a)

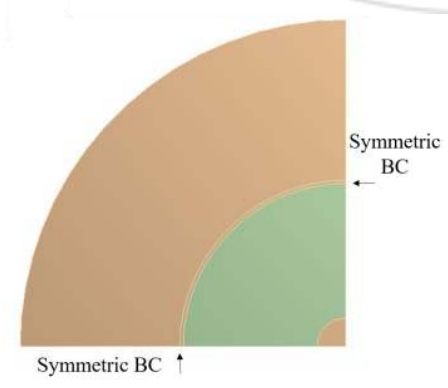

(b)
Figure 2: (a) Model Mesh, (b) Applied Symmetry Boundary Conditions

Contact pressure results were obtained using contact tool option in post processing. Results were taken at the central region of contact interface which shows approximately uniform pressure. Contact pressure was taken as the average contact pressure from nodal contour results.

In this study, FE models were analyzed for different contact formulations (Augmented Lagrange, Pure Penalty and Normal Lagrange) and contact stiffness factors (1, 10 and 100). In the second part of this study, results of contact offset based interference method were compared with geometry based interference method for the interference values of $5,10,15,25,35$ and $45 \mathrm{~mm}$.

\section{Results and Discussion}

Table 3 shows contact pressure obtained from FE model for different contact formulations compared with analytical calculation for the radial interference value of $10 \mathrm{~mm}$. In the similar way, table 4 and 5 tabulate contact pressure results for radial interference values of $20 \mathrm{~mm}$ and $40 \mathrm{~mm}$, respectively.

As seen in tables 3-5, analysis results match very closely with analytical calculations for different contact formulations in all analyzed cases though different contact formulations lead to different computational efficiencies as reflected by computation time.

Table 3: Contact Pressure (Radial Interference $=10 \mathrm{~mm}$ )

\begin{tabular}{|c|c|c|c|c|}
\hline Contact Formulation & $\begin{array}{c}\text { FE Model } \\
(\mathrm{MPa})\end{array}$ & $\begin{array}{c}\text { Analytical } \\
(\mathrm{MPa})\end{array}$ & $\%$ Diff & $\begin{array}{c}\text { CP Time } \\
(\mathrm{Sec})\end{array}$ \\
\hline Augmented Lagrange & 742.0 & 740.8 & $0.2 \%$ & 84.5 \\
\hline Pure Penalty (f=1) & 742.0 & 740.8 & $0.2 \%$ & 83.5 \\
\hline Pure Penalty (f=10) & 742.2 & 740.8 & $0.2 \%$ & 100.8 \\
\hline Pure Penalty (f=100) & 742.2 & 740.8 & $0.2 \%$ & 176.1 \\
\hline Normal Lagrange & 743.4 & 740.8 & $0.3 \%$ & 675.9 \\
\hline
\end{tabular}

Table 4: Contact Pressure (Radial Interference $=20 \mathrm{~mm}$ )

\begin{tabular}{|c|c|c|c|c|}
\hline Contact Formulation & $\begin{array}{c}\text { FE Model } \\
(\mathrm{MPa})\end{array}$ & $\begin{array}{c}\text { Analytical } \\
(\mathrm{MPa})\end{array}$ & $\%$ Diff & $\begin{array}{c}\text { CP Time } \\
(\mathrm{Sec})\end{array}$ \\
\hline Augmented Lagrange & 1469.0 & 1463.5 & $0.4 \%$ & 84.8 \\
\hline Pure Penalty (f=1) & 1469.0 & 1463.5 & $0.4 \%$ & 81.6 \\
\hline Pure Penalty (f=10) & 1469.1 & 1463.5 & $0.4 \%$ & 100.7 \\
\hline Pure Penalty (f=100) & 1468.8 & 1463.5 & $0.4 \%$ & 180.0 \\
\hline Normal Lagrange & 1472.8 & 1463.5 & $0.6 \%$ & 261.6 \\
\hline
\end{tabular}

Table 5: Contact Pressure (Radial Interference $=40 \mathrm{~mm}$ )

\begin{tabular}{|c|c|c|c|c|}
\hline Contact Formulation & $\begin{array}{c}\text { FE Model } \\
(\mathrm{MPa})\end{array}$ & $\begin{array}{c}\text { Analytical } \\
(\mathrm{MPa})\end{array}$ & $\%$ Diff & $\begin{array}{c}\text { CP Time } \\
(\mathrm{Sec})\end{array}$ \\
\hline Augmented Lagrange & 2879.9 & 2855.2 & $0.9 \%$ & 80.5 \\
\hline Pure Penalty (f=1) & 2879.9 & 2855.2 & $0.9 \%$ & 79.5 \\
\hline Pure Penalty (f=10) & 2878.0 & 2855.2 & $0.8 \%$ & 98.8 \\
\hline Pure Penalty (f=100) & 2876.3 & 2855.2 & $0.7 \%$ & 201.5 \\
\hline Normal Lagrange & 2890.8 & 2855.2 & $1.2 \%$ & 269.9 \\
\hline
\end{tabular}

As seen in tables, computational time is very close for Pure Penalty and Augmented Lagrange methods. As observed, computational time requirement for Pure Penalty method increases for higher contact stiffness factors.

Pure Penalty and Augmented Lagrange methods use penalty based formulations. In Pure Penalty method, contact force $(\mathrm{Fc})$ is related to contact stiffness (Kc) and penetration $(\mathrm{Xc})$ as per equation (2).

\section{Volume 5 Issue 11, November 2016 www.ijsr.net}




\section{International Journal of Science and Research (IJSR) \\ ISSN (Online): 2319-7064}

Index Copernicus Value (2013): 6.14 | Impact Factor (2015): 6.391

$$
\mathrm{Fc}=\mathrm{KcXc}
$$

In Augmented Lagrange method, extra Lagrangian term augments the pure penalty calculation as per equation (3)

$$
\mathrm{Fc}=\mathrm{KcXc}+\lambda
$$

At higher contact stiffness, higher computation time can be attributed to convergence difficulties to achieve lower penetration value.

Normal Lagrange computation time is much higher compared with penalty based methods. It can be explained based upon different method of enforcing contact compatibility in Normal Lagrange method compared with penalty based approaches. Normal Lagrange formulation treats contact pressure as an extra degree of freedom thus contact pressure is calculated explicitly as an extra DOF (degree of freedom).

In the second part of this study, contact pressure results were compared for contact offset based interference and geometry based interference methods with analytical calculations. Table 6 and Table 7 show comparative studies for Augmented Lagrange and Normal Lagrange formulations, respectively. As seen in Tables 6 and 7, contact offset based interference and geometry based interference methods provide close accuracy for smaller interference value.

Table 6: Contact Pressure Results (MPa): Geometrical Interference vs Contact Offset (Augmented Lagrange)

\begin{tabular}{|c|c|c|c|c|c|}
\hline Interference $(\mathrm{mm})$ & $\begin{array}{c}\text { Analytical } \\
\text { Calculation }\end{array}$ & $\begin{array}{c}\text { Result } \\
\text { (Geometrical Interference) }\end{array}$ & \% Variation & $\begin{array}{c}\text { Result } \\
\text { (Contact Offset) }\end{array}$ & $\%$ Variation \\
\hline 5 & 372.7 & 372.9 & $0.0 \%$ & 374.7 & $0.5 \%$ \\
\hline 10 & 740.8 & 742.0 & $0.2 \%$ & 749.4 & $1.2 \%$ \\
\hline 15 & 1104.4 & 1107.4 & $0.3 \%$ & 1124.0 & $1.8 \%$ \\
\hline 25 & 1818.1 & 1827.1 & $0.5 \%$ & 1872.6 & $3.0 \%$ \\
\hline 35 & 2513.9 & 2532.4 & $0.7 \%$ & 2620.3 & $4.2 \%$ \\
\hline 45 & 3192.2 & 3223.8 & $1.0 \%$ & 3366.4 & $5.5 \%$ \\
\hline
\end{tabular}

Table 7: Contact Pressure Results (MPa): Geometrical Interference vs Contact Offset (Normal Lagrange)

\begin{tabular}{|c|c|c|c|c|c|}
\hline Interference (mm) & $\begin{array}{c}\text { Analytical } \\
\text { Calculation }\end{array}$ & $\begin{array}{c}\text { Result (Geometrical } \\
\text { Interference) }\end{array}$ & \% Variation & $\begin{array}{c}\text { Result } \\
\text { (Contact Offset) }\end{array}$ & $\%$ Variation \\
\hline 5 & 372.7 & 373.3 & $0.2 \%$ & 374.4 & $0.4 \%$ \\
\hline 10 & 740.8 & 744.2 & $0.5 \%$ & 750.7 & $1.3 \%$ \\
\hline 15 & 1104.4 & 1109.8 & $0.5 \%$ & 1126.7 & $2.0 \%$ \\
\hline 25 & 1818.1 & 1832.8 & $0.8 \%$ & 1876.8 & $3.2 \%$ \\
\hline 35 & 2513.9 & 2541.7 & $1.1 \%$ & 2625.0 & $4.4 \%$ \\
\hline 45 & 3192.2 & 3236.8 & $1.4 \%$ & 3373.8 & $5.7 \%$ \\
\hline
\end{tabular}

As the value of the interference becomes higher, results of geometrical based interference are closer to analytical calculations than those of contact offset based method. It can be inferred that both methods can be used for simulating interference between parts though at higher interference values, geometry based interference method should be preferred to achieve better accuracy. Contact offset method is recommended for applications to make small adjustment only.

Contact based offset method has advantage that it does not require modification of geometry and mesh to simulate different interference values. In the same model, effect of different interference can be modeled by changing user defined contact offset value. Thus, comparative studies to understand the effect of different interference values on model behavior can be carried out with ease.

Studies in this work make use of simplified geometry since it allows comparison with analytical results though similar methodology can be used for more complex geometries and dissimilar materials.

\section{Conclusions}

Following conclusions can be drawn from the results of this study for the analyzed 3D Assembly cases.
- Interference analysis results are validated with analytical results for different contact formulations and contact stiffness values.

- Higher contact normal stiffness increases computational time.

- Computational time of Normal Lagrange method is much higher in comparison with those of penalty based methods.

- Results for geometry based interference and contact offset based interference methods match closely with analytical methods for lower interference values. At higher interference values, geometry based interference method provides better accuracy.

\section{References}

[1] R. Lewis, M. B. Marshall, R. S. Dwyer-Joyce, Measurement of Interface Pressure in Interference Fits, Journal of Mechanical Engineering Science Part C, 219 (2) (2005), 127-139

[2] F. Ozturk, Finite-element modelling of two-disc shrink fit assembly and an evaluation of material pairs of discs, Journal of Mechanical Engineering Science Part C, 225 (2) (2011), 263-273

[3] D. Benuzzi, G. Donzella, Prediction of the press-fit curve in the assembly of a railway axle and wheel, Journal of Rail and Rapid Transit, 218 (2004), 51-65

\section{Volume 5 Issue 11, November 2016}


[4] H. Boutoutaou, M. Bouaziz, J. F. Fontaine, Modeling of interference fits taking form defects of the surfaces in contact into account, Materials and Design, 32 (2011), 3692-3701

[5] G. M. Yang, J. C. Coquille, J. F. Fontaine, M. Lambertin, Influence of roughness on characteristic of tight interference fit of a shaft and hub, International Journal of Solids and Structures, 38 (2001), 7691-7701

[6] T. N. Chakherlou, M. Mirzajanzadeh, J. Vogwell, Experimental and numerical investigations into the effect of an interference fit on the fatigue life of double shear lap joints, Engineering Failure Analysis, 16 (2009), 2066-2080

[7] O. Eyercioglu, M. A. Kutuk, N. F. Yilmaz, Shrink fit design for precision gear forging dies, Journal of Materials Processing Technology, 209 (2009), 2186-2194

[8] B. You, Y. Luo, X. Wang, Contact algorithm of Finite Element analysis for prediction of press-fit curve, Journal of Information and Computational Science, 10 (9) (2013), 2591-2600

[9] J. E. Shigley, Mechanical Engineering Design, McGrawHill, New York, 1986.

\section{Author Profile}

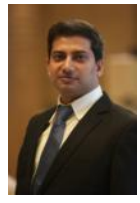

Bharat Panjwani received his B.Tech degree from IIT Kanpur, India and MEng degree from NUS, Singapore in Mechanical Engineering in 2002 and 2012, respectively. He has worked for Maruti Suzuki India Ltd, General Electric and John Deere in the past. He is currently associated with ANSYS, India as Senior Technology Specialist. He has expertise in the areas of non-linear structural analysis, dynamics and tribology. 